\title{
LOCATING MANDIBULAR FORAMEN IN CHILDREN OF DIFFERENT AGE GROUPS USING PANORAMIC RADIOGRAPHY
}

\author{
Hayri Akman, Koray Surme \\ Department of Pediatric Dentistry, Faculty of Dentistry, Alanya Aladdin Keykubat University, Alanya/Antalya, Turkey
}

\begin{abstract}
INTRODUCTION: Inferior alveolar nerve block (IANB) is the most widely used local anesthesia technique for mandibular primary or permanent teeth. The success of an adequate and effective nerve block with this technique depends on deposition of local anesthetic solution near mandibular foramen (MF), located on the medial side of mandibular ramus.

Овјестіves: The purpose of the study was to investigate the relative position of MF using digital panoramic radiographic technique to provide data for IANB technique in children.

MATERIAL AND METHODS: This retrospective study consisted of digital panoramic radiographs from 200 children, aged 5-14 years. Panoramic radiographs were divided into 5 groups according to Hellman dental classification. The distance of MF to occlusal plane, anterior border of the ramus, and lower border of the mandible were measured using computer software in different age groups, and then compared to determine the position of MF on radiographs. One-way analysis of variance test, which is one of the parametric tests, was used to compare mean values between the groups.

RESULTs: While MF was $0.85 \mathrm{~mm}$ above the occlusal plane in Hellman IIC age group, this distance increased with age progression, and there was a statistically significant difference between age groups. The distance from MF to anterior edge of the ramus and lower plane of the mandible increased in Hellman IVA age group, and there was a statistically significant difference between age groups.

ConcLusions: The position of MF in panoramic radiographs gradually changed posteriorly and upwards on the ramus with age progression.
\end{abstract}

KEY WORDS: mandibular foramen, inferior alveolar nerve block, panoramic radiography.

J Stoma 2021; 74, 3: 172-176

DOI: https://doi.org/10.5114/jos.2021.108837

\section{INTRODUCTION}

The control of pain and fear, which develops during dental procedures in children, is one of the most important issues of dental behavior management. Children who experience pain during restorative or surgical procedures may avoid dental treatment in the future $[1,2]$. Local anesthesia is a loss of sensation in a certain part of the body due to suppression of pain transmission in peripheral nerves or stimulating factor in nerve endings [3]. Intensive and effective local anesthesia facilitates a successful dental treatment of patients, especially pediatric ones, by alleviating anxiety and discomfort during restorative and surgical procedures [4].

\section{JOURNAL OF} STOMATOLOGY CZASOPISMO STOMATOLOGICZNE

AdDress for Correspondence: Dr. Koray Surme, Department of Pediatric Dentistry, Faculty of Dentistry, Alanya Aladdin Keykubat University, Alanya/Antalya, Turkey, e-mail: koray.surme@alanya.edu.tr 
The inferior alveolar nerve that penetrates the mandible through mandibular foramen (MF) located on medial surface of mandibular ramus, is responsible for sensory innervation of the lower gingiva and teeth as well as part of the lower lip, tongue, and chin within the ipsilateral side [5]. Inferior alveolar nerve block (IANB) is the most widely used local anesthesia technique for mandibular primary or permanent teeth [6]. The success of an adequate and effective nerve block with this technique depends on deposition of local anesthetic solution near the mandibular foramen, located in the medial side of mandibular ramus [7].

Successful IANB in pediatric patients is difficult to achieve, and the main reasons include additional branching of the nerve and, even more common, inappropriate needle insertion position $[8,9]$. Moreover, it has been reported that MF position changes as the child mature into adulthood, and dento-facial characteristics of patients with different ethnic groups may show a discrepancy [10]. Therefore, considering the change of MF position in individuals during the growth period can reduce the possibility of a failed injection [11].

Lower occlusal plane can be used in children as a reference to IANB [2]. Panoramic radiographs can help dentists in administering IANB by providing information regarding the positional relationship between lower occlusal plane and MF [12]. Various studies from different countries investigated MF location and its relationship with occlusal plane of deciduous molars in pediatric patients and provided inconsistent results (below, at the same level, or above) [13-16].

\section{OBJECTIVES}

The purpose of the present study was to investigate the relative position of MF using digital panoramic radiographic technique, to provide data for IANB technique in children, since there is lack of studies on the MF position on panoramic radiographs from different age groups in children.

\section{MATERIAL AND METHODS}

This retrospective study consisted of digital panoramic radiographs from 200 children, aged 5-14 years, who visited the Alanya Aladdin Keykubat University, Department of Pediatric Dentistry between January 2018 and March 2020 for dental treatments. All panoramic radiographs were taken during routine dental examinations, and no additional radiographs were provided for the current study. Moreover, all archived radiographs used in the study were obtained with ethical and deontological principles, including consents of the patients or their parents. After ethical approval from the institutional ethics committee, the subjects were randomly selected from a database of the institution, and were divided into five groups as per Hellman's dental developmental stages, with 40 children in each group (Table 1) [17]. Age and gender were also recorded.

All radiographs were taken by the same radiology technician, and all the subjects were positioned with their head oriented as per Frankfurt horizontal plane, parallel with earth horizontal plane. The lips of the subjects were positioned in a resting position, and the midline was adjusted correctly. Radiographs were taken using Kavo OP 3D (Instrumentarium Dental, PaloDEx Group, Tuusula, Finland), using $66 \mathrm{kV}, 5 \mathrm{~mA}$, and $12.8 \mathrm{~s}$ parameters. Evaluation and measurements of digital images were performed with Kavo CliniView ${ }^{\mathrm{TM}}$ (Instrumentarium Dental, PaloDEx Group, Tuusula, Finland) software, which automatically corrected magnification ratio of the panoramic radiography device on the images.

The radiographs used in the study have met the criteria of having an acceptable mandibular occlusal plane without any major anatomical abnormality. Radiographs with poor image quality, and patients presenting with a previous history of craniofacial trauma, temporomandibular joint, cranio-cervical disorders, orthodontic treatment, and in whom the mandibular occlusal plane was affected due to lack of posterior teeth and existence of immersed or supra-erupted mandibular teeth, were excluded from the study.

Here, right sides of the patients only were measured, as no difference between measurements made in the right and left regions was found in previous studies $[2,6]$. The location of MF was investigated radiographically by panoramic images, and the precise linear measurements were recorded using anatomic sites as reference points [11]. The analyzed points and planes were as follows (Figure 1):

- the points: point 1 - most superior anterior point of the mandibular canal; point 2 - most prominent point at the end of most fully erupted tooth; point 3 -

TABLE 1. Division of patients and their characteristics according to Hellman's dental development stages classification

\begin{tabular}{|l|c|c|c|}
\hline Group & Hellman's stages & Age (years) & Characteristics \\
\hline 1 & IIC & $5-7$ & Eruptive phase of the permanent first molars \\
\hline 2 & IIIA & $7-9$ & Completion of the eruption phase of permanent first molars \\
\hline 3 & IIIB & $9-12$ & Exchange phase of lateral incisor teeth \\
\hline 4 & IIIC & $11-12$ & Eruptive phase of the permanent second molars \\
\hline 5 & IVA & $12-13$ & Completion of the eruption phase of permanent second molars \\
\hline
\end{tabular}




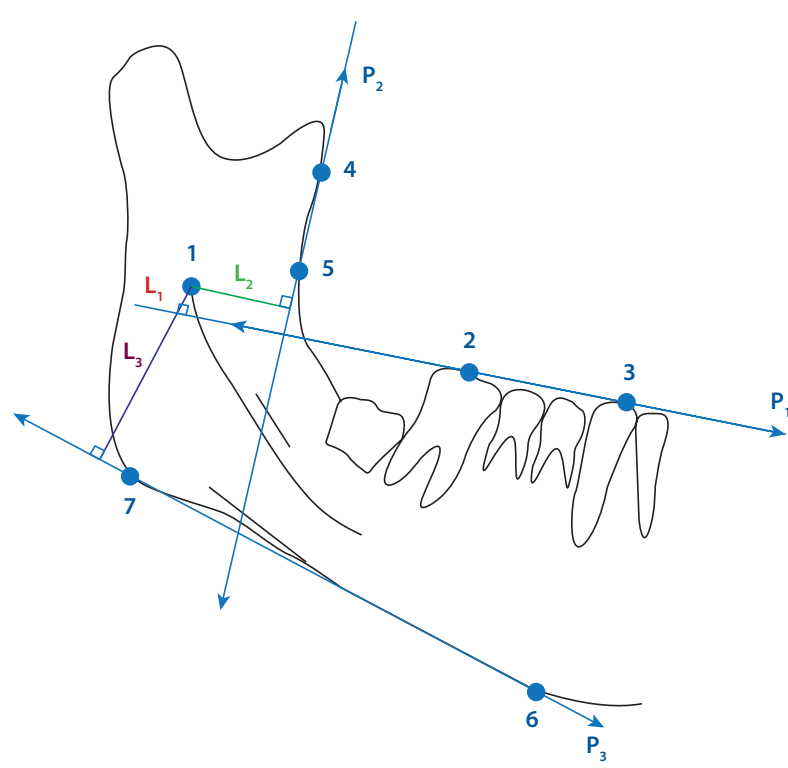

FIGURE 1. Reference points, planes, and measurements

most prominent point at the canine cusp tip; point $4-$ most prominent point of the anterior border of ramus; point 5 - deepest point of the anterior border of ramus; point 6 - most prominent inferior point of the body of mandible at canine area; point 7 - most prominent inferior point at the angle of the mandible.

- the planes: plane $1(\mathrm{P} 1)$ - occlusal plane connecting point 2 and point 3; plane 2 (P2) - plane connecting point 4 and point 5; plane 3 (P3) - plane connecting point 6 and point $7 ; \mathrm{L} 1$ - perpendicular line from point 1 to plane 1; L2 - perpendicular line from point 1 to plane 2; L3 - perpendicular line from point 1 to plane 3. All the measurements, including points, planes, and lines were drawn using a software with $100^{\text {th }}$ millimeter of accuracy, and registered. All the quantities were obtained by a single calibrated examiner, with five panoramic images daily in order to prevent any eye bias. To assess the reproducibility, 10 randomly selected panoramic radiographs were re-evaluated 2 weeks after being blindly assessed by the same researcher. Dahlberg's formula was used to determine method's error, and it was found to be within acceptable limits. Paired samples $t$-test was applied to test the significance of difference between the first and second measurements, with no statistically significant difference found between the measurements $(p>0.05)$.

In each developmental stage, mean and standard deviation values of all linear measurements based on the reference points were calculated using IBM SPSS 21.0 software (International Business Machines Corp., New York, USA) for data analysis. The significance level was set at $p<0.05$. Shapiro-Wilks' normality test and Levene's variance homogeneity test were applied to analyze distribution of the data, and it was observed that the data were distributed normally. Therefore, one-way analysis of variance test (as one of the parametric tests) was applied to compare mean values between the studied groups.

\section{RESULTS}

Panoramic radiographs of 200 subjects ( 97 boys and 103 girls) were included in the study. While the mean distance between MF and occlusal plane (L1) in the Hellman IIC age group was $0.85 \mathrm{~mm}$, this distance increased significantly in the IIIA age group, with its mean found as $2.27 \mathrm{~mm}$. L1 length increased significantly from the IIIC age group to the IVA age group, with a mean of $4.33 \mathrm{~mm}$ in the IVA age group. The mean length between MF and the ramus frontal plane (L2) was $14.35 \mathrm{~mm}$ in the IIC age group, and this length increased steadily towards the advanced age groups, showing $16.86 \mathrm{~mm}$ in the IVA age group. L2 length increased significantly from the IIIB age group to the IIIC age group. The mean length between $\mathrm{MF}$ and lower border of the mandible (L3) was $20.46 \mathrm{~mm}$ in the IIC age group. L3 length improved significantly in all age groups, with a mean of $27.63 \mathrm{~mm}$ in the IVA age group. The means and standard deviations of L1, L2, and L3 linear length measurements for each Hellman's dental development stage groups and the differences between age groups are presented in Table 2.

TABLE 2. Mean values and standard deviations of L1 (distance between mandibular foramen [MF] and occlusal plane), L2 (distance between MF and ramus frontal plane), and L3 (distance between MF and lower border of the mandible) measurements in all Hellman's dental developmental stages and $p$-values of ANOVA test

\begin{tabular}{|c|c|c|c|c|c|c|c|}
\hline \multirow[t]{2}{*}{ Age stages } & \multirow[t]{2}{*}{$n$} & \multicolumn{2}{|c|}{$\mathbf{L 1}$} & \multicolumn{2}{|c|}{ L2 } & \multicolumn{2}{|c|}{ L3 } \\
\hline & & Mean & SD & Mean & SD & Mean & SD \\
\hline$\| C$ & 40 & $0.85^{\mathrm{a}}$ & 1.61 & $14.35^{\mathrm{a}}$ & 1.67 & $20.46^{\mathrm{a}}$ & 1.85 \\
\hline IIIA & 40 & $2.27^{\mathrm{b}}$ & 0.77 & $14.95^{\mathrm{a}}$ & 1.95 & $21.65^{b}$ & 1.23 \\
\hline IIIB & 40 & $2.87^{\mathrm{bc}}$ & 0.55 & $15.10^{\mathrm{a}}$ & 1.82 & $23.57^{c}$ & 1.60 \\
\hline$\| I I C$ & 40 & $3.17 c$ & 1.00 & $16.16^{b}$ & 1.44 & $25.02^{d}$ & 1.26 \\
\hline IVA & 40 & $4.33^{d}$ & 1.54 & $16.86^{b}$ & 1.51 & $27.63^{e}$ & 1.88 \\
\hline$p$-values of ANOVA test & - & \multicolumn{2}{|c|}{$<0.001$} & \multicolumn{2}{|c|}{$<0.001$} & \multicolumn{2}{|c|}{$<0.001$} \\
\hline
\end{tabular}




\section{DISCUSSION}

One of the most commonly used anesthesia methods in dentistry is IANB, with a failure rate of $15-20 \%$. It is observed that the local anesthetic solution should be injected as close as possible to MF to increase the effectiveness of the mandibular block [2]. As the child grows, the bone growth and development, including the mandible, continues. Therefore, the position of MF constantly changes due to the mandible being in a constant remodeling phase $[8,9]$. Dentists are required to associate position changes in MF with dental development stages in order to provide effective anesthesia while applying IANB in children [6]. Trost et al. [18] reported that locating MF in panoramic radiographs can be easy application, providing valuable pre-operative information. Hence, this study was performed to investigate the positional alterations of MF from deciduous to permanent dentition according to Hellman's dental developmental stages using panoramic radiographs.

Previous studies have shown that there was no difference between male and female individuals in terms of the positional change of MF, and also no difference was found between measurements made in the right and left regions of the mandible $[2,6]$. For this reason, in this study, data belonging to both genders were pooled and evaluated. The data obtained from measurements taken from the right side of the mandible only were used; however, the teeth on both sides of the mandible were assessed to radiographically determine Hellman's dental developmental stage every patient.

The results of the present study revealed that the vertical measurement between MF and occlusal plane increased statistically significant as the child matures. It was determined that the localization of MF tended to move upward in the mandibular ramus with increasing age. This alteration may be related to the growth of the ramus itself [6]. Moreover, this result is consistent with findings of previous research $[2,6,8,19]$, where it was shown that the distance from MF to the alveolar crest plane or occlusal plane increased from stage IIC to IVA.

In a study of Benham [14], lateral cephalograms were examined, and it was found that MF was either at the same level as the occlusal plane or slightly above the occlusal plane during early mix dentition, and no statistically significant change in MF position until the age of 9 was observed. However, these results indicated that an increase in MF location as compared to occlusal plane was evident in the 9 to 11-year-old age group. On the other hand, several studies reported that vertical position of MF located slightly below occlusal plane in children under 8 years old, as contrary to the present study $[11,20]$. Avsar et al. [10] analyzed radiographic localization of mandibular anesthesia landmarks and, in opposition to the results of this study, did not observe an agerelated difference in the distance of MF from the occlusal plane. The reason for the different results of the above-mentioned studies could be methodologies used as well as various growth potential of the children due to their ethnic origins.

In studies examining antero-posterior positions of MF, localization of MF was defined as the distance between $\mathrm{MF}$ and the anterior border of the ramus greater than the distance between MF and the posterior border of the ramus $[2,6,9,11,19]$. However, previous studies reported that the antero-posterior position of MF does not change depending on age [14], and it is located in the midpoint of the ramus [21]. Also, there are studies reporting that horizontal localization of MF in the mandible ramus is more inferior-anterior in children compared to adults $[14,15,21]$. The results of the current study showed that the distance values between MF and the ramus frontal plane (L2) significantly increased from stage IIC to stage IVA, and MF was positioned more posteriorly on the mandible ramus in the horizontal direction. This result indicates that as the child matures, the anesthetic solution must be injected deeper into the ramus while performing IANB.

The measured vertical distance between the mandibular foramen and lower border of the mandible (L3) significantly increased about $7 \mathrm{~mm}$ from stage IIC to stage IVA, which is similar to previous studies' results $[2,6,9]$. It has been reported that vertical positional changes in MF may be related to child maturation, which results in the expansion of the ramus and bone formation at the lower border of the mandible $[6,21]$. In the present study, it was found that these changes were mostly specific between the stage IIIC and stage IVA groups, which may indicate that the mandible develops vertically more in the late mixed dentition stage than in the other stages.

When the findings from all these studies were evaluated, it was seen that the needle insertion point should be higher than the occlusal plane in children older than 7 years. Poonacha et al. conducted their study in Indian population, and reported that that the needle can be placed at the occlusal level in all age groups during IANB for successful inferior alveolar anesthesia [9]. However, it should be noted that MF may be below the occlusal plane in children under the age of 7 . In addition, IANB is performed with fully open-mouth technique, which may change the localization of MF [6].

The limitations of this study can involve panoramic radiographs taken within the presented methodology as well as the measurements obtained, which could not be as accurate as those acquired from three-dimensional computed tomography images. However, as stated in the literature, although panoramic radiographs are able to transform 3D information into 2D images, this imaging modality maintains acceptable morphological ratio levels and can be used for metric analysis [22]. In addition, the routine use of computed tomography in pediatric dentistry is limited, since the patient is exposed to radiation at higher levels than conventional radiographs during examinations with computed tomography [23]. 


\section{CONCLUSIONS}

Based on the outcomes of the present study, there was a statistically significant relationship between MF position and specific age ranges in the investigated children. It can be concluded that in child patients with stage IIC as per Hellman's dental developmental classification, MF was almost at the occlusion level, and its position gradually changed more posteriorly and upwardly on the ramus during children's maturation. This information could be helpful in guiding dentists to obtain more accurate and effective IANB in child patients.

\section{CONFLICT OF INTEREST}

The authors declare no potential conflicts of interest with respect to the research, authorship, and/or publication of this article.

\section{References}

1. Kot K, Krawczuk-Molęda E, Marek E, Lipski M. Self-inflicted injury as a complication following dental local anaesthesia in children - case reports. J Stomatol 2018; 71: 203-211.

2. Shukla RH, Tiku A. Correlation of mandibular foramen to occlusal plane as a clinical guide for inferior alveolar nerve block in children: a digital panoramic radiographic study. Contemp Clin Dent 2018; 9: 372-375.

3. Malamed SF (ed.). Handbook of Local Anesthesia. $6^{\text {th }}$ ed. St. Louis: Elsevier/Mosby; 2012.

4. Veneva ER, Belcheva AB. Local anesthesia in pediatric patients - a review of current and alternative methods, devices and techniques. Folia Medica 2018; 60: 381-388.

5. Pereira PN, Fernandes A, Gugisch RC, Zaroni FM, Franco A, Rebellato NLB. Radiographic assessment of the mandibular foramen in children: focus on anesthetic procedures. Arch Oral Res 2013; 9: 279-283.

6. Tsai HH. Panoramic radiographic findings of the mandibular foramen from deciduous to early permanent dentition. J Clin Pediatr Dent 2004; 28: 215-220.

7. Khoury J, Townsend G. Neural blockade anaesthesia of the mandibular nerve and its terminal branches: rationale for different anaesthetic techniques including their advantages and disadvantages. Anesthesiol Res Pract 2011; 2011: 307423.

8. Krishnamurthy NH, Unnikrishnan S, Ramachandra JA, Aral V. Evaluation of relative position of mandibular foramen in children as a reference for inferior alveolar nerve block using orthopantamograph. J Clin Diagn Res 2017; 11: 71-74.

9. Poonacha KS, Shigli AL, Indushekar KR. Relative position of the mandibular foramen in different age groups of children: a radiographic study. J Indian Soc Pedod Prev Dent 2010; 28: 173-178.

10. Afsar A, Haas DA, Rossouw PE, Wood RE. Radiographic localization of mandibular anesthesia landmarks. Oral Surg Oral Med Oral Pathol Oral Radiol Endod 1998; 86: 234-241.

11. Mohavved T, Makarem A, Imanimoghaddam M, Anbiaee N, Sarrafshirazi AR, Shakeri MT. Locating the mandibular foramen relative to the occlusal plane using panoramic radiography. J Appl Sci 2011; 11: 573-578.

12. Kositbowornchai S, Siritapetawee M, Damrongrungruang T, et al. Shape of the lingula and its localization by panoramic radiograph versus dry mandibular measurement. Surg Radiol Anat 2007; 29: 689-694.

13. Olsen NH. Anesthesia for the child patient. J Am Dent Assoc 1956; 53: 548-555.
14. Benham NR. The cephalometric position of the mandibular foramen with age. ASDC J Dent Child 1976; 43: 233-237.

15. Hwang TJ, Hsu SC, Huang QF, Guo MK. Age changes in location of mandibular foramen. Zhonghua Ya Yi Xue Hui Za Zhi 1990; 9: 98-103.

16. Bishara SE, Abdalla EM, Hoppens BJ. Cephalometric comparisons of dentofacial parameters between Egyptian and North American adolescents. Am J Orthod Dentofacial Orthop 1990; 97: 413-421.

17. Nakata M, Wei SH. Development of dental arch and occlusion. Occlusal Guidance in Pediatric Dentistry. $1^{\text {st }}$ ed. St. Louis, Tokyo: Ishiyaka Euro America, Inc.; 1986.

18. Trost O, Salignon V, Cheynel N, Malka G, Trouilloud P. A simple method to locate mandibular foramen: preliminary radiological study. Surg Radiol Anat 2010; 32: 927-931.

19. Kanno CM, de Oliveira JA, Cannon M, Carvalho AA. The mandibular lingula's position in children as a reference to inferior alveolar nerve block. J Dent Child 2005; 72: 56-60.

20. Pereira PN, Fernandes A, Gugisch RC, Zaroni FM, Franco A, Rebellato NLB. Radiographic assessment of the mandibular foramen in children: focus on anesthetic procedures. Arch Oral Res 2013; 9: 279-283.

21. Bennett CR. Monheim's Local Anesthesia and Pain Control in Dental Practices. $7^{\text {th }}$ ed. New Delhi: CBS Publishers; 1990.

22. Larheim TA, Svanaes DB. Reproducibility of rotational panoramic radiography: mandibular linear dimensions and angles. Am J Orthod Dentofacial Orthop 1986; 90: 45-51.

23. Miglioretti DL, Johnson E, Williams A, et al. The use of computed tomography in pediatrics and the associated radiation exposure and estimated cancer risk. JAMA Pediatr 2013; 167: 700-707. 